\title{
Acoustic Observatories for Ocean Tomography: Multi-Array Matched-Field Tomography
}

\author{
Cristiano Soares, Paulo Felisberto, Sérgio M. Jesus \\ Institute for Systems and Robotics \\ University of Algarve \\ 8005-139 Faro, Portugal \\ e-mail: $\{$ csoares, pfelis, sjesus\}@ualg.pt
}

\begin{abstract}
The present study proposes an MFT algorithm for directly estimating a two-dimensional range-dependent or three-dimensional temperature field. The acoustic system has multiple acoustic emitters and receiver arrays according to a given geometry. The acoustic signal is emitted from one emitter at a time, and collected at multiple receiver arrays. The MFT method is adapted to iteratively produce parameter estimates for each individual environmental cell. To accomplish this, an MF processor based on a multi-array acoustic data model is considered, in order to account for acoustic data collected simultaneously at several acoustic arrays.
\end{abstract}

\section{INTRODUCTION}

Ocean Acoustic Tomography (OAT) is a remote sensing technique that uses the propagation of sound waves for inferring environmental properties such as oceanographic quantities or geoacoustic parameters. The original idea consisted in using several acoustic emitters and receivers to observe the oceanographic quantities at relatively large scales (e.g. $1000 \mathrm{~km} \times$ $1000 \mathrm{~km}$ ), by comparing observed and predicted travel-times to infer sound-speed profiles and water column temperature [1], [2]. At the beginning of the 1990s, with the rapid increase in available computational power and the advent of very efficient computational techniques such as genetic algorithms and simulated annealing, a significant research effort was spent in shallow water for small scales (e.g. $20 \mathrm{~km} \times 20 \mathrm{~km}$ or less). Matched-Field Tomography (MFT), which is a generalization of Matched-Field Processing (MFP), has benefited with these developments, since it is computationally intensive. While MFP was originally developed for range-depth source localization, the MFT technique is applied when environmental properties, such as water column or seafloor parameters, are included in the inversion problem as unknowns.

Although OAT in shallow water has achieved an advanced degree of maturity, this technique has been mostly regarded as a remote sensing tool able to provide only integral properties of an ocean transect containing emitter and receiver. In fact, most experimental studies have considered only an emitterreceiver pair at a time, which implies that only a rangeaveraged value of each unknown parameter can be inferred, i.e., the underlying physical model is range-independent and a poor resolution is attained.

A few studies have considered concepts that aim at estimating the three-dimensional temperature field, or at least aim at using OAT to bring contributions to ocean temperature models, more as a technique for assimilation of acoustic data [3], [4], [5]. These studies present schemes involving an MFT inversion in a first step to estimate range-averaged sound speed profiles. In a second step, these profiles are merged to produce a three-dimensional field. In Refs. [3], [5] the second step is based on the division of the observed area in cells, and the contributions from several cross-sections is taken to estimate parameters in each individual cell. Refs. [3] deals with a deep water scenario where 3 or 4 vertical arrays spanning $1000 \mathrm{~m}$ of watercolumn are placed in the center of a gridded $250 \mathrm{~km}$ square and air-deployed shots are performed on the 4 edges of the square, where the proposed inversion method is an iterative MFP method using complex amplitude and phase. In Ref. [5] the second step is based on acoustic travel times of identifiable multipaths, which is difficult to be applied in shallow water experimental data due to complicated interaction with the boundaries. In Ref. [4] the sound-speed profiles are merged with direct point measurements of temperature and interpolated in order to yield an estimate of the sound-speed field.

The rise of ocean observatories across the planet, such as NEPTUNE, VENUS, ESONET/EMSO, among others, may become a new opportunity for a revival of tomographic concepts, specially for small-scales and medium-scales in littoral waters. These observatories are cabled networks designed to include many types of instrumentation and carry large amounts of data. For example, Neptune Canada [6], [7] has a backbone cable that carries both power and fiber-optic bidirectional communications across the network with repeaters that amplify the optical signal carrying information. The backbone contains branching units to distribute power and communications to spur cables connecting network nodes. The nodes work as an interface between the backbone and the junction boxes of local networks, providing communications to those networks with dedicated wavelengths, and converting power-supply from high-voltage into medium-voltage. The junction boxes convert medium-voltage into low-voltage to supply the instruments with energy and take care of the data communication by means of ethernet switches. These junction boxes may appear in parallel or in cascade.

Currently, ideas for including acoustics in ocean observatories are being proposed, as for example for marine-mammals tracking, underwater noise monitoring, environmental acoustic 
inversion, and acoustic communications. The idea is to use the implementation of such infra-structures as unprecedented opportunities for installing permanent acoustic instruments for carrying out activities in these fields, both in scientific and operational perspectives. The possibility of producing long data series, in the time-scale of years, opens important opportunities for developing and adapting existing or new tomographic concepts to an effective application in the observation of the ocean.

This study presents an MFT processing algorithm for directly estimating a two-dimensional range-dependent or threedimensional temperature field able to yield local estimates of a temperature field with spatial variability. This processing algorithm is designed for shallow water scenarios with emitter to receiver range up to $20 \mathrm{~km}$, and may find applicability in Acoustic Rapid Environmental Assessment (REA) or as a tomographic concept related to cabled Ocean Observatories. The acoustic system is a network of several nodes with acoustic emitters and receiver arrays according to a given geometry. The acoustic signal is emitted from one emitter at a time, and collected at multiple receiver arrays. Instead of following a gridding of the covered area, each acoustic path connecting an emitter to a receiver is divided into three environmental sections, and therefore a three-section rangedependent forward model is explicitly used. The environmental section containing the nodes are called anchor cells while environmental sections in the middle will be called middle cells. This is natural because the acoustic nodes are located at the extremities of the acoustic path, and each node may transmit to or receive from multiple other nodes, while in the middle no instruments exist.

In order to account for data received at multiple receiver arrays, an MF processor based on a multi-array acoustic data model is considered, meaning that each inversion uses acoustic data collected simultaneously at several acoustic arrays. The MFT method is iterative. The first iteration of each transect is based on range-independent forward models, and therefore a coarse estimate is obtained for each ocean transect. This estimate is used to obtain an initial estimate for the anchor cells in order to split the respective acoustic path into three environmental sections. From then on, the three section rangedependent forward models is considered in order to iteratively produce individual environmental estimates for each cell. This process is repeated until convergence is achieved.

\section{APPROACH}

The task of acoustically observing a three-dimensional environment requires an adequate acoustic network of emitters and receivers, and a processing scheme for acoustic data inversion properly designed for that acoustic network. The present section first proposes a minimal acoustic network, and then develops an iterative algorithm for the inversion of a temperature field, in a two-dimensional or three-dimensional shallow-water scenario.

\section{A. A minimal geometric configuration}

To perform three-dimensional tomography it requires the deployment of acoustic emitters and/or receivers at least three coordinates of the horizontal plane, which results in a triangle geometry. Figure 1 shows an example of a minimal network with a geometry seen in terms of the XY coordinates of an horizontal plane, for the emission and reception of acoustic signals in a three-dimensional space. This minimal geometry may allow for acoustically observing the environment traversed by the edges of the triangle - the acoustic propagation paths. The triangle vertices are labeled with letters A, B, C, and the acoustic paths indicated by arrows are labeled with labels $\mathrm{P}_{\mathrm{AB}}, \mathrm{P}_{\mathrm{BA}}, \mathrm{P}_{\mathrm{AC}}$, etc. Each arrow and respective label stands for a propagation direction. Since every vertex has an emitter and a receiver, each edge has two propagation paths, with opposite directions. Configurations like this, have been termed as $\mathrm{N}-2 \mathrm{D}$, or $2.5 \mathrm{D}$ rather than $3 \mathrm{D}$, because in fact only a multitude of range-depth planes, each one containing an emitter and a vertical array, can be effectively considered.

The next aspect to account for in the network is the division of the edges into segments, which can be seen as environmental sections or cells (depending on the paths that cross that area), represented in Fig. 1 by the red and green spots. The red spots have a triangular form in order to reflect the intention to consider that both segments sharing each vertex will be modeled with common physical properties. These areas are called anchor cells, because these cells are used to initialize the iterative search. Each anchor cell involve four acoustic paths. The green spots correspond to middle segments of the respective edges. Each middle segment is traversed by two acoustic paths. These segments are called middle cells. This is an ad-hoc configuration adapted to scales up to $20 \mathrm{~km}$ in shallow water with the objective to create portions of environment with local physical properties. It is acceptable to expect that the present configuration enables the observability of the local environmental properties within each cell since each acoustic path will traverse the cell by the maximum available length, and each acoustic inversion will take place with acoustic data collected at multiple receiver arrays. As the MFT technique is based on the uniqueness of the acoustic field with regard to the underlying environmental properties, the joint processing of acoustic data collected with two or more arrays should increase the joint field uniqueness by significantly increasing the total number of available acoustic receivers, or by increasing the diversity of the acoustic fields traveling across diverse environmental media. It also appear acceptable that for the scales often considered in shallow water three environmental sections allow for a reasonable environmental discrimination. As will be developed below, for example, to estimate parameters in anchor cells, one can take at least field data collected from the two receiver arrays contained in the opposite acoustic nodes.

As the proposed approach has a geometric nature, and a potentially high complexity, specially if more instrumentation nodes are included, it is a good practice to implement a 


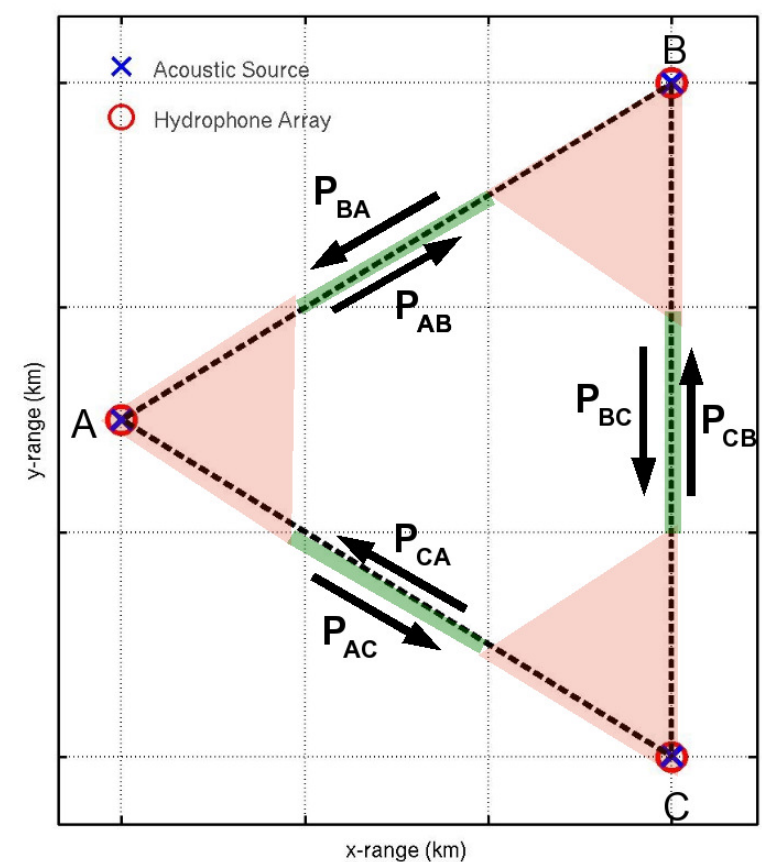

Fig. 1. Triangular acoustic configuration. The acoustic instruments are placed at the vertices of the triangle, which are connected by propagation paths. The red areas indicate the anchor environmental cells, and the red areas indicate middle environmental cells. The arrows indicate acoustic propagation direction.

systematic description of the geometric setup. This is useful for computer code implementations, in order to directly reflect the acoustic deployment and to allow for easy inclusion or exclusion of instrumentation nodes and acoustic paths, or more generally, in order, to deal with complex problems requiring splitting up the geometric setup into smaller less complex configurations. The geometric setup is described on the basis of three types of objects:

- Instrumentation nodes. First all nodes containing instruments are defined. For now it is assumed that every node has both an acoustic emitter and a vertical receiver array. Each node is parameterized with the following parameters: horizontal plane coordinates $X$ and $Y$; source depth $Z_{\mathrm{s}}$; the receiver array is described with deepest receiver depth $Z_{\mathrm{r}}$, receiver spacing $\delta Z$, and receiver number $N_{\mathrm{r}}$. Only uniformly spaced vertical arrays are considered.

- Acoustic paths. An acoustic path is a straight line with origin at a node with an acoustic emitter and destination at a node with an acoustic receiver array. Thus, an acoustic path is described solely by indicating an origin node $O$ and a destination node $D$. Note that for each propagation direction a path is considered. For example, to connect two nodes, each having both emitter and receivers, two paths are created.

- Environmental cells. An environmental cell is an area of the horizontal plane crossed by one or multiple acoustic paths, and its description includes the indication of every acoustic path crossing it. A cell is described by the following parameters: cell number to uniquely identify each cell; list of acoustic paths crossing the cell and respective starting range, for indicating the segment interval on that path; a list of acoustic paths considered for forward computation in the inversion problem (not all acoustic path will necessarily enter the acoustic inversion of a given cell); cell type: this parameter can assume values $\mathbf{A}$, which stands for anchor, and $\mathbf{M}$, which stands for middle; a cell is also described by environmental parameters, such as bathymetry, geoacoustic parameters, and soundspeed profile. This completes the geometric description of the acoustic network.

This type of geometric description serves as input to the inversion problem, and allows for a systematic description and re-configuration of the network, even in cases where a large number of acoustic nodes come into play, as for example, when a moving acoustic source is to be taken into account.

\section{B. The acoustic inversion algorithm}

This section develops an iterative MFT algorithm for the acoustic inversion of parameters of environmental cells set over an horizontal plane and with acoustic instruments, emitters and receivers, deployed over a three dimensional space. This iterative algorithm builds on the triangle configuration represented in Fig. 1 without loss of generality as it can be directly adapted to more complex architectures.

The development of this algorithm consists mainly on establishing a set of cost functions $P$ that compare the observed field with replica fields generated for hypothetical parameter vectors, that are maximized in order to yield parameter estimates for a specific environmental cell, in terms of their dependencies on acoustic data and intermediate parameter estimates. A parameter vector is denoted by the Greek letter $\theta$ with a superscript indicating the iteration number, and one or two subscript letters that identify the corresponding cell: anchor cells are identified with single letters A, B, and C; middle cells will be identified with pairs of letters $A B, B C$, and $\mathrm{AC}$, specifying the adjacent anchor cells. The acoustic data involved in each estimator is specified by $Y$ with two superscript letters, in order to specify the acoustic path, i.e., emitter and receivers involved, as for example $Y^{\mathrm{AB}}$, which refers to acoustic data obtained with emitter at position $\mathrm{A}$ and receiver array at position $\mathrm{B}$. The algorithm consists of three phases, where each phase has a respective type of estimator:

- Phase 1 is an initialization iteration in order to obtain a coarse estimate on the anchor cell parameters.

- Phase 2 is to estimate parameters of middle environmental cells.

- Phase 3 is to estimate parameters of anchor environmental cells.

When the algorithm is started (iteration 1), no estimates are available. In this particular situation the segmentation 
of each acoustic path into three environmental sections can not apply, since no parameter values are available to assign to individual sections sharing the same acoustic path. Thus, the algorithm naturally proceeds with a range-independent physical model. Phase 1 is set up to initialize the algorithm with an acoustic inversion for the parameter set of each anchor cell. The estimators for the anchor cells are given as:

$$
\begin{aligned}
& \underline{\hat{\theta}}_{\mathrm{A}}^{1}=\arg \max _{\underline{\theta}} P\left(\underline{\theta}, Y^{\mathrm{AB}}, Y^{\mathrm{AC}}\right) \\
& \underline{\hat{\theta}}_{\mathrm{B}}^{1}=\arg \max _{\underline{\theta}} P\left(\underline{\theta}, Y^{\mathrm{BA}}, Y^{\mathrm{BC}}\right) \\
& \underline{\hat{\theta}}_{\mathrm{C}}^{1}=\arg \max _{\underline{\theta}} P\left(\underline{\theta}, Y^{\mathrm{CA}}, Y^{\mathrm{CB}}\right),
\end{aligned}
$$

which states that for each anchor cell the inversion is based on the transmission from that cell to the receiver arrays of the other two anchor cells, and the only parameter vector involved in this step is an hypothetical value with respect to the whole range of both acoustic paths. The superscript 1 indicates that this set is applied only in the first iteration. Each estimated parameter vector is an integral value over both acoustic path departing from a common node, and is therefore based on a different pair of acoustic paths. The purpose of this processing step is to seek for a solution that is an alternative to the physical reality, but still providing a minimal degree of acoustical adjustment, in order to allow a meaningful representation of the reality. These initial estimates should reflect the environment spanned by the respective acoustic paths. Once this set of estimates has been obtained, the algorithm goes into Phase 2.

Phase 2 is set up for the inversion of parameters in the middle sections. This phase uses the acoustic field crossing the environmental section in both directions, and the parameter estimates of adjacent anchor cells, yielded either from Phase 1 , if it is the second iteration, or Phase 3, otherwise. The estimators for the middle cells are given as:

$$
\begin{gathered}
\underline{\hat{\theta}}_{\mathrm{AB}}^{n}=\arg \max _{\underline{\theta}} P\left(\underline{\theta}, Y^{\mathrm{AB}}, Y^{\mathrm{BA}}, \underline{\hat{\theta}}_{\mathrm{A}}^{n-1}, \underline{\hat{\theta}}_{\mathrm{B}}^{n-1}\right) \\
\underline{\hat{\theta}}_{\mathrm{BC}}^{n}=\arg \max _{\underline{\theta}} P\left(\underline{\theta}, Y^{\mathrm{BC}}, Y^{\mathrm{CB}}, \underline{\hat{\theta}}_{\mathrm{B}}^{n-1}, \underline{\hat{\theta}}_{\mathrm{C}}^{n-1}\right) \\
\underline{\hat{\theta}}_{\mathrm{AC}}^{n}=\arg \max _{\underline{\theta}} P\left(\underline{\theta}, Y^{\mathrm{AC}}, Y^{\mathrm{CA}}, \underline{\hat{\theta}}_{\mathrm{A}}^{n-1}, \underline{\hat{\theta}}_{\mathrm{C}}^{n-1},\right)
\end{gathered}
$$

for $n>1$. Note that the expression on the left hand of eq. (2c), the superscript ${ }^{n}$ denotes the current iteration, and on the right hand the superscript ${ }^{n-1}$ denotes the previous iteration, which may be Phase 1 or Phase 3, carried out to yield estimates on parameter vectors of the adjacent cells. In Phase 2, the threeenvironmental sections physical model is used for forward modelling. Once this set of estimates is obtained, the algorithm proceeds with Phase 3.

Phase 3 is set up to provide estimates on the anchor cells. In comparison to Phase 1 the differences are the inclusion of the parameter estimates obtained in the previous iteration run in Phase 2, and therefore a three environmental sections physical model is used in Phase 3. The estimators for the anchor cells in Phase 3 are given as:

$$
\begin{aligned}
& \underline{\hat{\theta}}_{\mathrm{A}}^{n}=\arg \max _{\underline{\theta}} P\left(\underline{\theta}, Y^{\mathrm{AB}}, Y^{\mathrm{AC}}, \underline{\hat{\theta}}_{\mathrm{AB}}^{n-1}, \underline{\hat{\theta}}_{\mathrm{A}}^{n-1}, \underline{\hat{\theta}}_{\mathrm{AC}}^{n-1}, \underline{\hat{\theta}}_{\mathrm{C}}^{n-1}\right) \\
& \underline{\hat{\theta}}_{\mathrm{B}}^{n}=\arg \max _{\underline{\theta}} P\left(\underline{\theta}, Y^{\mathrm{BA}}, Y^{\mathrm{BC}}, \underline{\hat{\theta}}_{\mathrm{AB}}^{n-1}, \underline{\hat{\theta}}_{\mathrm{A}}^{n}, \underline{\hat{\theta}}_{\mathrm{BC}}^{n-1}, \underline{\hat{\theta}}_{\mathrm{C}}^{n-1}\right) \\
& \hat{\theta}_{\mathrm{C}}^{n}=\arg \max _{\underline{\theta}} P\left(\underline{\theta}, Y^{\mathrm{AC}}, Y^{\mathrm{CA}}, \underline{\hat{\theta}}_{\mathrm{AC}}^{n-1}, \underline{\hat{\theta}}_{\mathrm{A}}^{n}, \underline{\hat{\theta}}_{\mathrm{BC}}^{n-1}, \underline{\hat{\theta}}_{\mathrm{B}}^{n}\right)
\end{aligned}
$$

for $n>2$. In this case, estimators $\underline{\hat{\theta}}_{\mathrm{B}}^{n}$ and $\underline{\hat{\theta}}_{\mathrm{C}}^{n}$ take into account parameter estimates of the current iteration. After this set of estimators, the algorithm proceeds back to Phase 2. The sequence will be Phase 1, Phase 2, Phase 3, Phase 2, Phase 3, ..., until full convergence is achieved. In practice, it requires the definition of a criterion to decide that convergence has been achieved in order to terminate the search. In this study, the search is terminated if the complete parameter set remains unchanged for 2 iterations.

This inversion method is computationally intensive for several factors. It takes a number $N_{\text {it }}$ of iterations until the search is interrupted. In the case of the triangle configuration, each iteration is composed of three inversions, one for each cell, at any Phase. The parameter search interval is divided into $Q$ quantization steps, which equals the number of model evaluations required for each inversion, in the case of an exhaustive search. For each hypothetical solution two forward models are computed. During iteration 1, a range-independent environmental model is used, and after, all forward computations use a three-environmental sections model. The number of forward models computed for a complete inversion is given by $3 \times 2 \times Q \times N_{\text {it }}$.

There are some remarks that need to be made at this point. First, it is required that the range-independent model in Phase 1 can yield a replica field with a minimal degree of adjustment to the observed acoustic fields in order to allow the iterative search procedure to proceed on a trajectory allowing convergence to a solution compatible with the real physical scenario. A similar concept was exploited in the focalization processor, whose aim was to allow range-depth source localization with an alternative physical model [8]. Since, in past studies successful acoustic modeling with a range-independent model was achieved with source-receiver ranges of up to $10 \mathrm{~km}$ [9], it is acceptable to initialize the algorithm with the hypothesis in Phase 1. Another remark is related to the geometry of the acoustic system: in order to increase the field diversity, one should deploy emitters and/or receivers at different depths, and set up acoustic paths with different lengths.

\section{Data model and matched-field processor}

The three-dimensional acoustic inversion algorithm uses a set of parameter estimators that is the maximization of cost functions taking into account acoustic data collected simultaneously at 2 (or more) acoustic arrays. To derive the functional used in the parameter estimator a multi-array data 
model is therefore required. The data model considered herein is an extension of the broadband data model discussed in Ref. [10], which is a concatenation of single frequency components of the signal observed across a vertical array. In the present case the idea is to include frequency components of a signal observed across two or more vertical arrays. The linear data model can be written as

$$
\underline{Y}=\mathbf{H A} \underline{S}+\underline{N}
$$

where $\underline{Y}=\left[\underline{Y}_{1}^{T}\left(\omega_{1}\right) \underline{Y}_{2}^{T}\left(\omega_{1}\right) \underline{Y}_{1}^{T}\left(\omega_{2}\right) \underline{Y}_{2}^{T}\left(\omega_{2}\right)\right]^{T}$. For each frequency all receiver arrays are contiguously concatenated. The vector $\underline{Y}_{p}^{T}\left(\omega_{k}\right)$ represents the $k^{\text {th }}$ frequency component observed at the receivers of the $p^{\text {th }}$ vertical array. In the case of two arrays and two frequencies, i.e., $P=2$, and $K=2$, the channel matrix is given as

$\mathbf{H}=\left[\begin{array}{cccc}\underline{H}_{1}\left(\omega_{1}, \underline{\theta}_{1}\right) & \underline{0} & \underline{0} & \underline{0} \\ \underline{0} & \underline{H}_{2}\left(\omega_{1}, \underline{\theta}_{2}\right) & \underline{0} & \underline{0} \\ \underline{0} & \underline{0} & \underline{H}_{1}\left(\omega_{2}, \underline{\theta}_{1}\right) & \underline{0} \\ \underline{0} & \underline{0} & \underline{0} & \underline{H}_{2}\left(\omega_{2}, \underline{\theta}_{2}\right)\end{array}\right]$,

where $\underline{H}_{p}\left(\omega_{k}, \underline{\theta}_{p}\right)$ is a vector representing the acoustic response respecting to the $p^{\text {th }}$ vertical array, and $\underline{\theta}_{p}$ is the parameter vector for the respective acoustic propagation path. Matrix A contains random perturbation factors $\alpha_{p}\left(\omega_{k}\right)$, that aims at modeling random features that can not be accounted by the acoustic propagation model. From the matrix arrangement of the data model, it follows that

$$
\mathbf{A}=\left[\begin{array}{cc}
\alpha_{1}\left(\omega_{1}\right) & 0 \\
\alpha_{2}\left(\omega_{1}\right) & 0 \\
0 & \alpha_{1}\left(\omega_{2}\right) \\
0 & \alpha_{2}\left(\omega_{2}\right)
\end{array}\right]
$$

where $\alpha_{p}\left(\omega_{k}\right)$ the perturbation coefficients for the field received at the $p^{\text {th }}$ array and the $k^{\text {th }}$ frequency. A complete analysis on this data model would hypothesize on cross-correlations combining arrays and frequencies, such as $\mathrm{E}\left[\alpha_{p_{1}}\left(\omega_{k_{1}}\right) \alpha_{p_{2}}\left(\omega_{k_{2}}\right)\right]$. This analysis is out of the scope of this paper. Finally, the signal vector has the following construction

$$
\underline{S}=\left[\begin{array}{l}
S\left(\omega_{1}\right) \\
S\left(\omega_{2}\right)
\end{array}\right],
$$

and the noise vector $\underline{N}$ is a concatenation of noise vectors $\underline{N}_{p}\left(\omega_{k}\right)$, which are assumed Gaussian zero mean and spatially uncorrelated.

As mentioned above the remaining task in terms of modeling consists in hypothesizing on the cross-frequency and crossarray second order statistics. This is expressed by means of the spectral density matrix (SDM) which is the second order statistics given as

$$
\begin{aligned}
\mathbf{C}_{Y Y} & =\mathrm{E}\left[\underline{Y Y}{ }^{H}\right] \\
& =\mathbf{H E}\left[\mathbf{A} \underline{S} \underline{S}^{H} \mathbf{A}^{H}\right] \mathbf{H}^{H}+\mathrm{E}\left[\underline{N} \underline{N}^{H}\right],
\end{aligned}
$$

where $\mathrm{E}[]$ denotes mathematical expectation, and ${ }^{H}$ denotes conjugate transpose. The $\operatorname{SDM} \mathbf{C}_{Y Y}$ has dimensions $K \mathcal{L} \times$
$K \mathcal{L}$, where $\mathcal{L}$ is the total number of receivers at the $P$ arrays. This matrix consists of block SDMs

$$
\mathbf{C}_{Y Y, p_{1}, p_{2}}\left(\omega_{k_{1}}, \omega_{k_{2}}\right)=\mathrm{E}\left[\underline{Y}_{p_{1}}\left(\omega_{k_{1}}\right) \underline{Y}_{p_{2}}^{H}\left(\omega_{k_{2}}\right)\right],
$$

for $p_{1}, p_{2}=1, \ldots, P$, and $k_{1}, k_{2}=1, \ldots, K$. In order to compute the cross-correlation matrix of the concatenation vector $\underline{Y}$, it is necessary to associate $\underline{\tilde{S}}=\mathbf{A} \underline{S}$, and then

$$
\begin{aligned}
\mathbf{C}_{Y Y} & =\mathbf{H E}\left[\underline{\tilde{S}} \underline{\tilde{S}}^{H}\right] \mathbf{H}^{H}+\mathrm{E}\left[\underline{N} \underline{N}^{H}\right] \\
& =\mathbf{H C}_{S S} \mathbf{H}^{H}+\sigma_{N}^{2} \mathbf{C}_{N N},
\end{aligned}
$$

where $\mathbf{C}_{S S}$ is the signal matrix including the second-order statistics accounting both for the emitted signals and the perturbation factors.

The parameter estimators described in section II-B use the broadband Bartlett processor [10] as the functional for comparison of the replica fields with the observed field based on the multi-array data model:

$$
P(\underline{\theta})=\frac{\operatorname{tr}\left[\mathbf{H}^{H}(\underline{\theta}) \mathbf{C}_{Y Y} \mathbf{H}(\underline{\theta}) \mathbf{C}_{S S}\right]}{\operatorname{tr}\left[\mathbf{H}^{H}(\underline{\theta}) \mathbf{C}_{N N} \mathbf{H}(\underline{\theta}) \mathbf{C}_{S S}\right]} .
$$

In order to account for signals propagating across different acoustic paths are incoherent, the signal matrix $\mathbf{C}_{S S}$ is made zero off diagonal.

\section{Simulations}

This section presents examples of application in order to infer on the viability of the iterative three-dimensional MFT algorithm. The algorithm is applied to a parameter estimation scenario with only one unknown parameter which is an EOF coefficient that presents variability over the horizontal XY plane. Both the geometric setup and the estimation procedure follows exactly what is presented in section II, including the environmental sectioning of the acoustic propagation paths. Two cases are inspected: first, in order to test the convergence to the true parameter solution, a simple case where the synthetic data is generated with the unknown parameter constant across each environmental cell considered in the geometric model; then, in a more realistic situation, the synthetic data is generated with the unknown parameter as an arbitrary function of the coordinate in the horizontal plane of the threedimensional space.

The geometric set up is the following: the acoustic nodes containing acoustic emitter and receiver array are placed at every vertex of an equilateral triangle, with sidelengths of $15 \mathrm{~km}$. The instrumentation setup is detailed in Table I, where for each triangle vertex, A, B, C, XY coordinates, emitter and receiver depth are indicated. The receiver depth is with respect to the deepest acoustic receiver of each array. The vertical array has an aperture of $60 \mathrm{~m}$, and 16 uniformly spaced receiver elements.

The ocean transect is modeled as a three-layer model, with a $120 \mathrm{~m}$ watercolumn, a sediment layer, and an infinite subbottom. The water column temperature is modeled as

$$
t(z)=\bar{t}(z)+\alpha \operatorname{EOF} 1(z)
$$




\begin{tabular}{|c|c|c|c|c|}
\hline Vertex & $\begin{array}{c}\text { x coordinate } \\
(\mathrm{km})\end{array}$ & $\begin{array}{c}\text { y coordinate } \\
(\mathrm{km})\end{array}$ & $\begin{array}{c}\text { Source depth } \\
(\mathrm{m})\end{array}$ & $\begin{array}{c}\text { Receiver depth } \\
(\mathrm{m})\end{array}$ \\
\hline \hline $\mathrm{A}$ & 0.00 & 0.00 & 50 & 66 \\
\hline $\mathrm{B}$ & 12.99 & 7.50 & 60 & 76 \\
\hline $\mathrm{C}$ & 12.99 & -7.50 & 70 & 86 \\
\hline
\end{tabular}

TABLE I

DESCRIPTION OF INSTRUMENT NODES A, B, C. RECEIVER DEPTH RESPECTS TO THE DEEPEST RECEIVER OF THE ARRAY. (a)

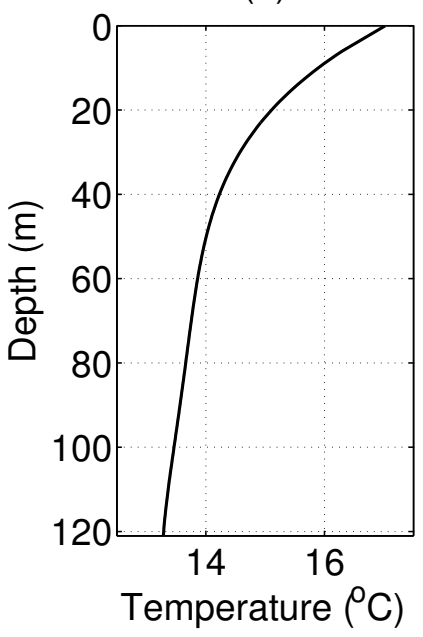

(b)

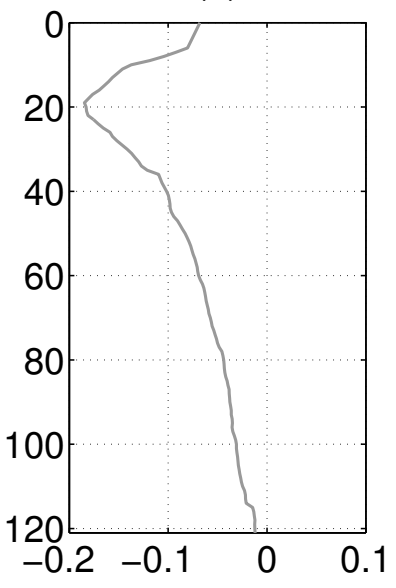

Fig. 2. Temperature data used in forward modeling: (a) mean temperature profile; (b) first EOF. The temperature data was taken during the RADAR'07 sea trial.

where $\bar{t}(z)$ is the mean profile and $\operatorname{EOF} 1(z)$ is the first EOF of a set of historical data. The EOF coefficient $\alpha$ is the amplitude of a temperature perturbation. Figure 2 shows the temperature data used in this study, which was obtained from measurements taken during the RADAR'07 sea trial [11]. For soundspeed computations, a mean salinity profile from the same data set with an average value of $35.9 \mathrm{ppt}$ was used. The acoustic field is simulated for a single frequency of $1000 \mathrm{~Hz}$ using the C-SNAP normal modes code to compute forward models [12]. The cross-correlation matrix is generated according to eq. (9), with a diagonal signal matrix, and with noise power equal zero. Acoustic field data is generated for 6 acoustic paths, two for each edge of the triangle.

\section{A. Case 1: range-independent environmental sections}

In the first case of synthetic data the environmental segments in the red and green areas of the triangle in Fig. 1 are assumed to be range independent. The segments in the red area (anchor cells) have an unperturbed temperature profile, i.e., the temperature profile is the mean temperature shown in Fig. 2, and the segments in green areas (middle cells) have a perturbation, whose amount is given by the amplitude of $\alpha$ in eq. (10), which is equal for all middle cells. Simulations were carried out for increasing values of the perturbation amplitude, in order to conclude on the convergence to the true perturbation value. Table II summarizes the inversion results

\begin{tabular}{|c|c|c|}
\hline $\begin{array}{c}\text { True parameter } \\
\alpha_{\mathrm{AC}}\end{array}$ & Iterations & Error \\
\hline \hline-0.5 & 4 & 0 \\
\hline-1 & 6 & 0 \\
\hline-2 & 6 & 0.17 \\
\hline-3 & 12 & 0.22 \\
\hline-4 & never & - \\
\hline
\end{tabular}

TABLE II

SUMMARY OF SIMULATION RESULTS FOR PARAMETER $\alpha_{\text {AC }}$ OF ENVIRONMENTAL CELL AC: TRUE VALUES OF THE PARAMETER (LEFT COLUMN); NUMBER OF ITERATIONS FOR CONVERGENCE (MIDDLE COLUMN); FINAL ESTIMATION ERROR (RIGHT COLUMN).

obtained for the middle cell $\mathrm{AC}$ for increasing temperature perturbation in the middle cell, with $\alpha$ ranging from -0.5 to -4. It shows that up to a certain amount of temperature perturbation the iterative inversion algorithm is able to converge to the exact solution, with an error of zero (for all environmental cells). However, beyond a certain threshold this is not true anymore: for moderate cases the iterative estimation algorithm will converge to an alternative solution presenting a relatively small error. For heavy perturbations, the algorithm enters into a cyclic divergence. This happens due to the model mismatch in the first iteration that is run in Phase 1. As explained above, Phase 1 uses a range-independent physical model for the estimation of the anchor cells' parameters. The result of the first iteration is only a temporary solution in order to allow for an initialization of the algorithm, since from iteration 2 on, run on Phase 2, previous parameter estimates are required to enter the inversion. In the present single parameter inversion it just happens that the iterative algorithm enters into a trajectory that does not allow to attain the true parameters, even with perfect physical and statistical modeling, and from which it can not escape if no additional degrees of freedom are allowed. This is a drawback caused by the estimators' interdependency on parameter cells with common acoustic paths. Figure 3 shows the MF response as a function of the unknown parameter for the environmental segments on acoustic path $\mathrm{AB}$, the first and second iterations, and for the convergence iteration, for $\alpha=-3$. Panels (a) and (c) respectively show the MF response for anchor cells $\mathrm{A}$ and $\mathrm{B}$, while panel (b) is for the middle cell $\mathrm{AB}$. The true value of each cell is marked with a stem terminated with circle. In iteration 1 the MF response is maximized with a range-independent forward model, which clearly is a physical mismatch situation, as the Bartlett function exhibits significantly weak maxima away from the true value. Iteration 2 is for cell $\mathrm{AB}$ with initial parameter estimates for cells $\mathrm{A}$ and $B$ available. This inversion already uses the range-dependent three-sector model, but is biased by the estimation error of iteration 1. Iterations 3 and 4 significantly step towards the true parameter values in all cells, and the maximum MF responses are obtained in iterations 11 and 12, however without attaining the true parameter. 
(a)

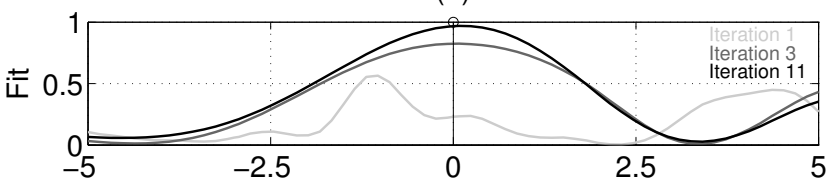

(b)

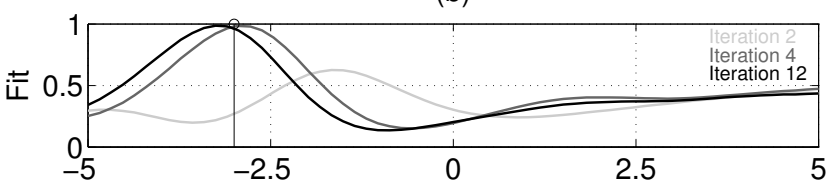

(c)

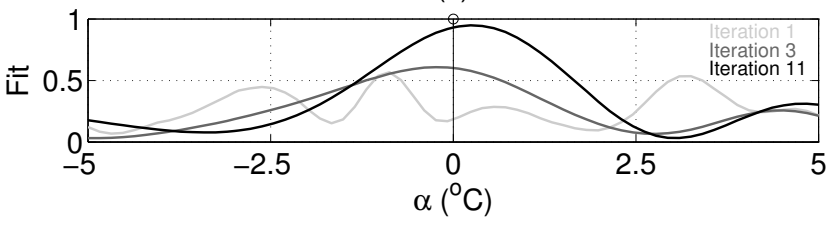

Fig. 3. Bartlett functions for inversions in acoustic path AB: (a) anchor cell $\mathrm{A}$; (b) middle cell AB; (c) anchor cell B. The stem terminated with circle indicates the true parameter value $(\alpha=-3)$.

\section{B. Case 2: range-dependent environmental sections}

This section presents a simulation case where the watercolumn temperature features a more realistic distribution as it is modeled with an EOF coefficient that varies smoothly over the horizontal plane XY. Figure 4 shows the EOF coefficient as a function of $x$ and $y$ ranges together with the acoustic setup. All settings presented for Case 1 were preserved in Case 2, and therefore the model used during the inversion is no longer in exact agreement with the forward model used to generate the data. For synthetic data generation, a onedimensional EOF coefficient $\alpha$ was extracted by interpolation across each triangle edge with a resolution of $1 \mathrm{~km}$.

Figure 5 shows the MF response as a function of the unknown parameter for the cells on the $\mathrm{AB}$ acoustic path, where panel (a) is for parameter $\alpha_{\mathrm{A}}$, panel (b) is for parameter $\alpha_{\mathrm{AB}}$, and panel (c) is for parameter $\alpha_{\mathrm{B}}$. The search has converged after 6 iterations only. These plots illustrate the ability of this inversion algorithm to precisely converge to an unambiguous parameter estimate close to the true value, although range-independent environmental cells are considered for the forward modelling. The MF response is close to maximum. Table III compares the true parameter value with the parameter estimate for all cells, reinforcing this observation. The inversion algorithm has allowed to obtain a good discrimination of the average parameter value measured across each environmental cell.

\section{CONCLusions}

This paper presents a method for three-dimensional inversion of acoustic data. This is based on a minimal acoustic network of three nodes each containing both an emitter and a receiver array. This allows to perform acoustic transmissions from each node to the other two. Each pair of nodes is connected by an acoustic path, which is divided into three

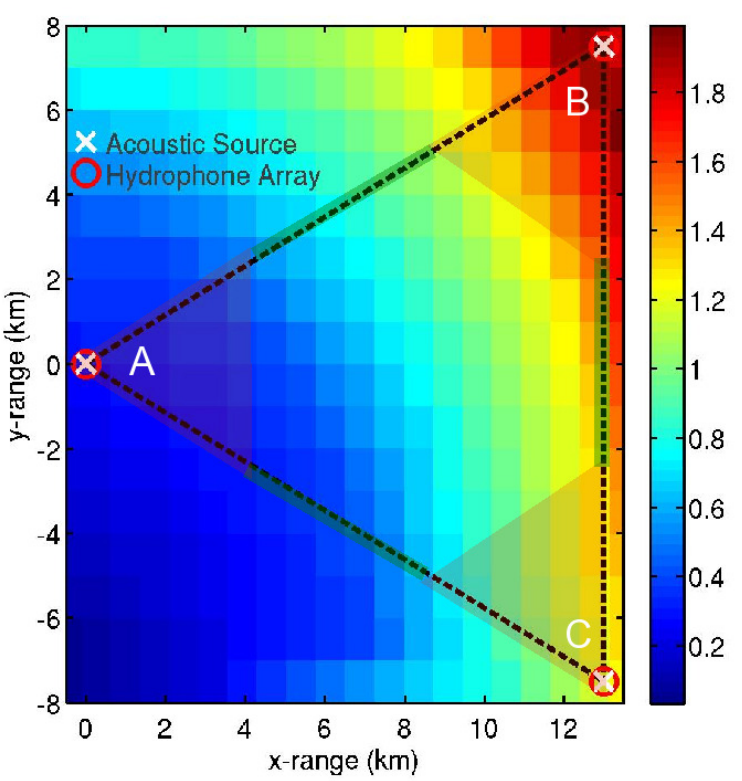

Fig. 4. Two-dimensional EOF coefficient generated for acoustic data simulation.

(a)

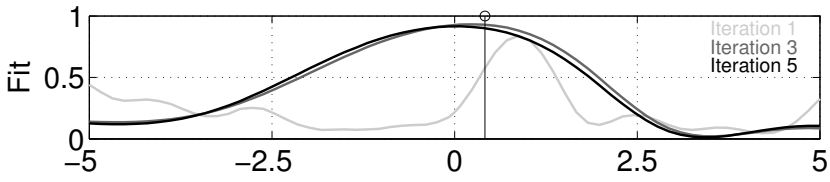

(b)

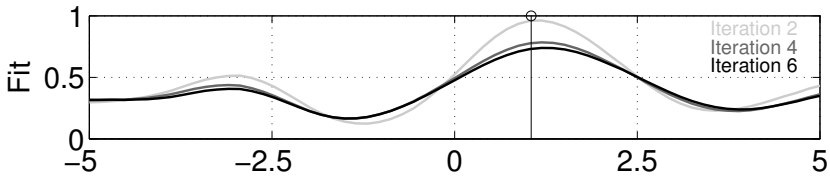

(c)

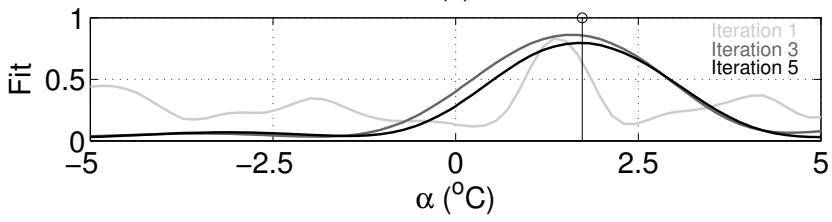

Fig. 5. Bartlett functions for inversions in acoustic path $\mathrm{AB}$ : (a) anchor cell A; (b) middle cell AB; (c) anchor cell $\mathrm{B}$. The stem terminated with circle indicates the true parameter value.

TABLE III

SUMMARY OF SIMULATION RESULTS FOR ALL ENVIRONMENTAL CELLS: THE TRUE VALUE OF EACH CELL IS TAKEN AS THE MEAN VALUE OF THE EOF COEFFICIENT OVER THE RESPECTIVE SEGMENT.

\begin{tabular}{|c|c|c|}
\hline & & \\
Parameter & True & Estimate \\
\hline \hline$\alpha_{A}$ & 0.4148 & 0.0794 \\
\hline$\alpha_{A B}$ & 1.0483 & 1.1905 \\
\hline$\alpha_{B}$ & 1.7334 & 1.6667 \\
\hline$\alpha_{A C}$ & 0.4631 & 0.2381 \\
\hline$\alpha_{C}$ & 1.1301 & 1.1905 \\
\hline$\alpha_{B C}$ & 1.5774 & 1.8254 \\
\hline
\end{tabular}


environmental sections. The inversion is performed by means of an iterative algorithm based on an MFT processor that estimates the environmental parameters of each environmental cell at a time using intermediate estimates of adjacent cells until convergence is achieved.

The algorithm was tested in a case where the geometric configuration is a triangle with three acoustic nodes, resulting in six individual cells, and the unknowns are an EOF coefficient of a temperature model for each. The results indicate that under this configuration the unknown parameter of all cells can be unambiguously observed by acoustic means, and that convergence to the true value can be achieved if a meaningful parameter estimate can be obtained in the first iteration.

Future work shall investigate on the feasibility of this approach to more realistic cases as acoustic configurations with only receiving or only emitting nodes, as for example the case of a moving acoustic source, and extend the application of this approach to more realistic multi-parameter inversion problems.

\section{ACKNOWLEDGMENT}

This work was partially funded by ESONET - European Seas Observatory Network, Contract no.: 036851. We would like to thank Professor Michael Taroudakis, University of Crete, Greece, for leading the Acoustic Tomography Group created in the scope of the ESONET Project.

\section{REFERENCES}

[1] P. F. Worcester and R. C. Spindel. North pacific acoustic laboratory. J. Acoust. Soc. Am., 117(3):1499-1510, 2005.

[2] J.L. Spiesberger, D.E. Frye, J. O'Brien, H. Hurlburt, J.W. McCaffrey, M. Johnson, and J. Kenny. Global acoustic mapping of ocean temperatures (gamot). In OCEANS '93. Engineering in Harmony with Ocean. Proceedings, pages I253 -I257 vol.1, October 1993.

[3] A. Tolstoy, O. Diachok, and N. L. Frazer. Acoustic tomography via matched field processing. J. Acoust. Soc. America, 89(3):1119-1127, March 1991.

[4] P. Elisseeff, H. Schmidt, M. Johnson, D. Herold, N. R. Chapman, and M. M. McDonald. Acoustic tomography of a coastal front in haro strait, british columbia. The Journal of the Acoustical Society of America, 106(1):169-184, 1999.

[5] P. Felisberto and S. M. Jesus. An hybrid acoustic-oceanographic method for estimating the spatial distribution of sound-speed. In S. M. Jesus and O. C. Rodrguez, editors, Proc. European Conf. on Underwater Acoustics (ECUA) 2006, pages 663-668, June 2006.

[6] S.T. Lentz. The neptune canada communications network. In OCEANS 2007, pages $1-5$, October 2007.

[7] P. Phibbs and S. Lentz. The implementation of the neptune canada backbone network. In OCEANS 2007, pages 1 -5, October 2007.

[8] M. D. Collins and W. A. Kuperman. Focalization: Environmental focusing and source localization. J. Acoust. Soc. America, 90:1410 1422, 1991.

[9] C. Soares, M. Siderius, and S. M. Jesus. Source localization in a time-varying ocean waveguide. J. Acoust. Soc. Am., 112(5):1879-1889, November 2002.

[10] Cristiano Soares, Sérgio M. Jesus, and Emanuel Coelho. Environmental inversion using high-resolution matched-field processing. The Journal of the Acoustical Society of America, 122(6):3391-3404, 2007.

[11] C. Soares, S. M. Jesus, P. Hursky, T. Folegot, C. Martins, F. Zabel, L. Quaresma, Dong-Shan Ko, and E. F. Coelho. Random array of drifting acoustic receivers (radar'07). Technical Report Rep. 04/07, SiPLAB Report, University of Algarve, December 2007.

[12] C. M. Ferla, M. B. Porter, and F. B. Jensen. C-SNAP: Coupled SACLANTCEN normal mode propagation loss model. Memorandum SM-274, SACLANTCEN Undersea Research Center, La Spezia, Italy, 1993. 